\title{
Static and dynamic balance in young athletes
}

\author{
LEONARDO RICOTTI
}

Biorobotics Institute - Scuola Superiore Sant'Anna, Pontedera (Pisa), Italy

\begin{abstract}
Ricotti L. Static and dynamic balance in young athletes. J. Hum. Sport Exerc. Vol. 6, No. 4, pp. 616628,2011 . The creation of a mature athlete necessarily passes through the expression of his potential during each phase of his development. Young athletes' trainers often neglect specific balance programs, above all in certain sports (e.g. soccer) where balance is poorly considered, but that is fundamental for the execution of complex technical movements, as well as for the prevention of future injuries. In this paper we highlight that balance training at specific ages is important for the maturation of the sensorimotor abilities that are important for a high level athlete. We discuss the main methods used to assess balance in sports, including some useful formulae that can be used to quantify postural performances, and we report the major findings concerning static and dynamic analyses on children and adolescents. Finally, we discuss the existing literature regarding balance recordings on adults, adolescents and children involved in one or more sport activities at regional, national or international level, highlighting the need of further research on the mechanisms underlying balance improvements related to different sport activities at various ages. Keywords: STATIC BALANCE, DYNAMIC BALANCE, COP, CHILDREN TRAINING, YOUNG ATHLETES, POSTURAL PERFORMANCES.
\end{abstract}

Corresponding author. Biorobotics Institute - Scuola Superiore Sant'Anna. Viale Rinaldo Piaggio 34, 56025 Pontedera (Pisa). Italy

E-mail: I.ricotti@sssup.it

Submitted for publication July 2011

Accepted for publication December 2011

JOURNAL OF HUMAN SPORT \& EXERCISE ISSN 1988-5202

(C) Faculty of Education. University of Alicante

doi:10.4100/jhse.2011.64.05 


\section{INTRODUCTION}

Children and adolescents are not "small adults". Their body and their mentality are quantitatively but also qualitatively different from the adult ones, and they must be therefore considered not just smaller, but different. They are subjected to a maturation process that is not linear, but characterized by "growing spikes", affecting their capability to learn particular motor skills. An effective training program for children and adolescents must take into account the psycho-physical particularities of each age range, in order to focus on and to exploit at the maximum level the specific age-related motor learning abilities. Weineck (2001) described the main age ranges for children and adolescents, specifying the sensibility of each age to the learning of a specific motor skill (Table 1).

Table 1. Main age ranges of children and adolescents, and associated motor skills that are particularly sensible to be learnt from children of corresponding ages. $M$ refers to males, $F$ to females.

\begin{tabular}{ccc}
\hline Age & Chronological age (years) & Sensible trainable motor skills \\
\hline First childhood & $0-1$ & - \\
Childhood & $1-3$ & - \\
Pre-scholar age & $3-6 / 7$ & Motor creativity \\
First scholar age & $6 / 7-10$ & Basic technical skills \\
First pubertal age & F: $11 / 12-13 / 14$ & Balance, \\
& M: $12 / 13-14 / 15$ & Coordination \\
Adolescence & F: $13 / 14-17 / 18$ & Coordination, Conditional abilities \\
& M: $14 / 15-18 / 19$ & (force, resistance, velocity) \\
Adult age & F: $17 / 18$ & Conditional abilities (force, resistance, \\
& M: $18 / 19$ & velocity) \\
\hline
\end{tabular}

From Table 1 it is evident that balance and coordination emerge as key trainable motor skills from the age of 10 , raising the attention on the importance of specific equilibrium and coordination-based training sessions at that age.

Coordinative abilities (dexterity) rely on the movement control and regulation processes, and they are fundamental in sports, as they allow athletes to easily control their own motor actions. Moreover, they allow the learning of complex movements in a relatively rapid way. One of the main components of coordinative abilities is balance. Postural control (or balance) can be defined statically as the ability to maintain a base of support with minimal movement, and dynamically as the ability to perform a task while maintaining a stable position (Winter et al., 1990). This ability is influenced by a complexity of factors, that are sensory information (from somatosensory, visual and vestibular systems), joint range of motion (ROM), and strength (Grigg, 1994; Nasher et al., 1982; Palmieri et al., 2003; Palmieri et al., 2002), and it is responsible for the correct execution of complex sport movements, as well as for protection against injuries. It has been demonstrated that injuries to the knee and ankle are common in today's athletes, and they are most prevalent in cutting and jumping sports such as volleyball, football, soccer and basketball (Griffin, 2000). Non-contact mechanisms, such as landing from a jump, frequently lead to joint or ligament injuries, that are probably the result of strength deficits or impaired stability and balance (Wikstrom et al., 2004). Some literature evidence suggests that a superior balance among experienced athletes is the result of repetitive training experiences that influence motor responses (Balter et al., 2004); others argue that superior balance is the result of training experience 
influencing a person's ability to attend to relevant proprioceptive and visual cues (Ashton-Miller et al., 2001). Research therefore suggests that changes in both sensory and motor systems influence balance performance, and such changes seem to be more effective if induced in children, during the appropriate age ranges, by means of specific training, as suggested by (Weineck, 2001). The assessment and the periodic monitoring of static and dynamic balance in young athletes can be an important instrument in order to correctly define and change training programs, taking into account the sport practiced, the rate of improvement in balance scores over time and the athlete's age. This would allow to maximize, in each period of the athlete's body development, the harmonic creation and optimization of a wide set of fundamental motor abilities.

In the present review we describe the methods used in the literature and in sporting practice to quantitatively measure static and dynamic balance, particularly focusing on center-of-pressure recordings and their use as clinical as well as sport performance indexes. Then, we focus on the literature data regarding balance measurements in healthy and non-healthy children, and we finally try to summarize the several reasons for which balance monitoring and improvement is important in young athletes performing different sports. In the conclusions and perspectives, we remark the need for further studies on static and dynamic balance of children performing different sports, and we propose some ideas in order to expand the existing literature in this field.

\section{METHODS FOR ASSESSING BALANCE IN SPORTS}

There are several postural control strategies identified in literature to maintain balance in a variety of static and dynamic activity circumstances. The ankle strategy, for example, (based on the restoration of stability through body movement centred around the ankle joint) is mainly used when the equilibrium perturbation is small and the support surface is firm. The hip strategy is used in correspondence to larger perturbations, as the ankle strategy does not provide enough force to maintain postural stability; the movement is focused at the hip joint. When the perturbation is large enough to displace the centre of gravity outside the person's base of support, the stepping or hopping strategy is used to restore balance. Muscle synergies are used to adapt these postural strategies to a variety of dynamic situations (Emery, 2003). Whatever is the strategy to keep or to restore it, balance can be quantified by means of several indexes, related to different tests. Some of these are used both in clinical and in sport trials, to assess respectively the presence of a pathology and an athlete's balance performance level after a specific training program, and all of them can be used to assess balance of children. The selection of a suitable method generally depends on the goals and results aimed at, as there is no single assessment technique that could be used as a true indicator of the overall integrity and functionality of the balance control system (Winter et al., 1990). For all the existing tests, the selection of measuring time and stance conditions is essential. In typical force platform measurements, for example, the most frequently used duration is from 20 to 30 seconds, even if longer and shorter times have also been used (Table 2).

Table 2. Example of different testing times used for static and dynamic balance tests on children and adolescents.

\begin{tabular}{cccc}
\hline $\begin{array}{c}\text { Type of balance } \\
\text { test(s) performed }\end{array}$ & Age of participants & Test duration & Reference(s) \\
\hline Static & $6-10$ years & $10 \mathrm{~s}$ & (Rival, 2005) \\
Static and Dynamic & $14-17$ years & $20 \mathrm{~s}$ & (Ribaldi, 1987) \\
Static balance test & $5-6$ years & $30 \mathrm{~s}$ & (Kirshenbaum, 2001) \\
Static and Dynamic & 14 years & $35 \mathrm{~s}$ & (Mallau, 2007) \\
\hline
\end{tabular}


The measuring time should be long enough to provide an appreciable result, but short enough to avoid fatigue with the measurements (Iverson et al., 1990). Variations in the technique of measuring posture during stance must be also considered; standing on two legs is the easiest position, but the subject can be asked to stand with the feet together, touching the heel to the toe (tandem stance) or standing on one leg (Harrison, 1994).

Regarding static balance, traditional tests to evaluate it consist in measuring the length of time subjects can maintain a particular equilibrium position (Graybiel, 1966). In such tests, visual inputs are often minimised, in order to not interfere with the assessment of balance control, that is dependent on the vestibular and somatosensory functions. Static balance in athletes has traditionally been assessed with the standing Romberg test; the subject stands with the feet together, arms at the side, and eyes closed. A tendency to sway or fall to one side is considered to indicate a loss of proprioception (Booher \& Thibodeau, 1995). This test has been strongly criticized for its lack of sensitivity and objectivity (Guskiewicz \& Perrin, 1996). The Balance Error Scoring System (BESS), described by Riemann et al. (Riemann et al., 1999) involves different stance positions on stable and unstable surfaces for the dominant and non-dominant limbs. Each position is held with eyes closed and hands on hips for a certain amount of time (for example 30 seconds), and scoring is determined by recording of errors. Errors include opening eyes, lifting hands from hip, touchdown of non-stance foot, step, hop, or other movement of the stance foot or feet, lifting forefoot or heel, moving hip into more than $30^{\circ}$ of flexion or abduction, remaining out of position for longer than 5 seconds.

More recently, advancements in technology have provided the scientific community with force platform systems for the quantitative assessment of static balance. These systems provide an easy, practical, and cost-effective method to quantitatively assess functional balance through the analysis of postural sway. The most common systems are the Chattecx Balance System, the EquiTest, the Force Plate, the Pro Balance Master, and the Smart Balance Master (Guskiewicz \& Perrin, 1996). Such systems basically record the displacements of the centre of foot pressure (COP) by means of strain gauge sensors embedded in the platform structure.

The movements of the COP reflect both the horizontal location of the centre-of-gravity (COG) and the ground reaction forces due to the muscular activity of the lower limb, transmitted through the foot. They are therefore related to the subject's body movements during the stance, named "body sway" (Era et al., 1996). More precisely, body sway is defined as the persistent oscillation of the centre of mass (COM) referring to the antero-posterior (AP) and medio-lateral (ML) axes. This variable is not usually measured in a direct way (for example by means of kinematic analysis with joint markers), but it has been demonstrated that COP recordings from a force platform can be used to compute a subject's COM (Morasso et al., 1999). Data processing is used in order to compute selected parameters of total body sway from the time series of COP positions. Typical parameters in platform measurements are the mean COP position (as a reference point), the anterior-posterior and the lateral sway (Kinney et al., 1997), the length of the sway path (Juntunen et al., 1987), the sway velocity (Hytönen et al., 1993) and the sway area (Ekdahl et al., 1989). COP values from a force platform can be measured under variable visual and surface conditions, depending on which experimental conditions are more appropriate for the purpose of the study.

The reliability of platform measurements has been reported in literature as high (Ishizaki et al., 1991), even if some authors report about the poor reliability of platform measurements (Huxham et al., 2001), and about the low reliability coefficients of COP measurements between test occasions (Hill et al., 1996). The differences among authors' conclusions could lie in differences in the methods and populations used for their studies. 
Regarding dynamic balance, a simple test to measure it is the Star Excursion Balance Test (SEBT) (Gribble \& Hertel, 1999). It requires participants to maintain a single-leg stable stance and to reach for maximal distance with the other leg in 8 different directions. When the stability of the supporting leg is compromised, the test is repeated. Data have to be normalized to the subject's leg length. As for static balance, the need for a higher objectivity and reliability of measures, together with the corresponding advances in technology, make the force platforms' measurements the most used method, in literature, for assessing dynamic balance in adults and in children.

The classic protocol followed to assess dynamic balance using a force platform is the jump protocol. The subject is asked to stand on the platform maintaining a double-leg stance, then to jump (at a height which varies depending on the purpose of the experiment) and to land on one foot, immediately putting the hands on hips and trying to recover a stable stance as quickly as possible, while facing straight ahead. Many parameters can be extracted from force platform recordings during the execution of this task. One of the most used is the Time To Stabilization (TTS), a measure of neuromuscular control that incorporates both sensory and mechanical systems to master the complex task of jump landing (Wikstrom, 2004). The vertical TTS is usually calculated as the time when the vertical force component reaches and remains within $5.0 \%$ of the subject's body weight after landing (McKinley \& Pedotti, 1992); the AP and ML TTS are usually determined by means of sequential estimation (Colby, 1999): this technique incorporates an algorithm to calculate a cumulative average of the data points in a series by successively adding 1 point at a time. This cumulative average is then compared against the overall series mean, and a subject is considered stable when the sequential average remains within \pm 0.25 standard deviations of the overall series mean. In 2005 a new parameter was introduced, named Dynamic Postural Stability Index (DPSI) (Wikstrom, 2005). It is also based on the jump protocol, and it takes into account the fluctuations of the COM around the equilibrium point during the time after landing, represented by the corresponding COP fluctuations in 3 different directions (ML, AP, and vertical). This leads to the computation of 3 different stability indexes: the medio-lateral stability index (MLSI), the anterior-posterior stability index (APSI), and the vertical stability index (VSI), calculated as follows.

$$
\begin{aligned}
& \text { MLSI }=\sqrt{\left(\sum(0-x)^{2} / \text { number.of.data.points }\right)} \\
& A P S I=\sqrt{\left(\sum(0-y)^{2} / \text { number.of.data.points }\right)} \\
& V S I=\sqrt{\left(\sum(\text { body.weight }-z)^{2} /\right. \text { number.of .data.points }}
\end{aligned}
$$

The variables $x, y$, and $z$ refer to the components of the force platform signal along the corresponding axis during time. The DPSI is a composite of the 3 parameters described before, and it is sensitive to changes in all 3 directions.

$$
D P S I=\sqrt{\left(\sum(0-x)^{2}+\sum(0-y)^{2}+\sum(\text { body.weight }-z)^{2} /\right. \text { number.of .data.points }}
$$

The DPSI is an index of how fast a subject can recover a stable stance after a dynamic task (the jump, in this case). DPSI values are normally evaluated over different time intervals (for example 3,5 and 10 seconds after jump landing), to check the decrease of the parameter (and the consequent recover of stability) over time. 
Dynamic balance conditions have also been investigated by means of seesaw devices, having only 1 degree of free motion and generating instability, laid on the force platform. In this case, it is also possible to investigate the COP excursion in the frequency domain, to assess the preferential involvement of short or long neuronal loops in balance regulation (Maki, 1986; Peterka, 2002). Fast Fourier transforms can be applied to COP displacements from 0 to $20 \mathrm{~Hz}$, and the total spectral energy calculated and distributed in 3 frequency bands: low frequencies $(0-0.5 \mathrm{~Hz})$, medium frequencies $(0.5-$ $2.0 \mathrm{~Hz}$ ), and high frequencies (greater than $2 \mathrm{~Hz}$ ).It has been shown that low frequencies mostly account for visuovestibular regulation (Dichgans, 1976; Nagy, 2004), medium frequencies for cerebellar regulation (Njiokiktjien, 1978) and high frequencies for proprioceptive regulation (Gurfinkel, 1973). In contrast with force plate systems, the Biodex Stability System (BSS) uses a circular platform that is free to move in the AP and ML axes simultaneously. The stability of the platform can be also varied, by varying the preset resistance force applied by springs on the underside of the platform (Arnold \& Schmitz, 1998). Rather than measuring the deviation of the COP during static conditions, this device measures the degree of tilt about each axis during dynamic conditions. Thus, the BBS appears to provide more specific information on ankle joint movements.

\section{CHILDREN, ADOLESCENTS, AND BALANCE}

From an exquisitely neuroscientific point of view, an ontogenetic model of the development of balance control in children was described in 1998 (Assaiante, 1998), hypothesizing that the two main modes of equilibrium control (ascending and descending temporal organization) operate alternatively.

Reliability reports and normative data for stance assessment in elementary school children were limited in literature before 2000. Children's development of postural stability using bilateral force plate measurements was described in some studies before 2006 (Cambier, 2001; Hatzitaki, 2002; Lebiedowska \& Syczewska, 2000; Lee, 2004; Nolan \& Grigorenko, 2005; Rival, 2005; Schmid, 2005), but none of these works incorporated reliability reports on the evaluation of the method of using force plate measurements for a childhood population. Interesting conclusions can be however taken from such studies, particularly from (Schmid, 2005), focusing on 148 children with ages ranging from 7 to 11 . They showed that the role of vision varies within the studied age range, and that the maturation of balance control is not complete, even at the age of 11 .

In 2006 a study based on assessing static and dynamic balance on 99 children from 9 to 10 years old, including test-retest reliability, with the aim of furnishing reference values for the balance at that age was published (Geldhof, 2006). The results showed significantly lower sway velocities in girls compared to boys, indicating a better postural control in girls at age 9 to 10 years.

Together with such a general approach to children balance, research also focuses on specific pathologies-related balance disturbs, in order to deeply understand how a pathology influences the development of postural control of children, but also to find some diagnostic parameters based on balance screening. Donker et al. in 2008 compared postural sway fluctuations of children with cerebral palsy (CP) and of healthy, typically developing (TD) children (Donker et al., 2008). They also investigated the effect of visual information on postural sway in the previously mentioned groups. The findings of this study indicated that postural control in CP children is deteriorated compared to TD children, and visual deprivation influenced sway characteristics differently in the two groups.

Regarding adolescents, the literature reports investigations on their static and dynamic balance, mainly in relation with pathologies or injuries. The pioneering work of Ribadi et al. compared three groups of adolescents, composed of sighted, blindfolded, and congenitally blind students respectively (Ribaldi et al., 1987). Significant differences were found in all the three groups, with the sighted group demonstrating superior balance for both measures, and with the blind group showing significantly better 
performances with respect to the sighted blindfolded group only concerning dynamic balance. The influence of adolescence overweight and of previous wrist bone fractures on static and dynamic balance was investigated in 2003 by Gouldinga et al. (Gouldinga et al., 2003). Overweight was found to be a negative factor for balance performances, while fracture history did not affect balance measurements. Similar studies were performed on adolescents affected by scoliosis (Mallau et al., 2007), autism and Asperger syndrome (Freitag et al., 2007), and by epilepsy (Kowulski, 1995).

\section{THE IMPORTANCE OF BALANCE MONITORING AND IMPROVEMENT IN YOUNG ATHLETES}

Proper balance control in the achievement of motor skills is mainly based on muscular synergies that minimise the displacements of the centre of gravity. This constitutes the base for the correct execution of complex technical gestures, as well as for the minimization of injury risks. There are many examples in literature regarding the evaluation of static and dynamic balance of adult athletes, competing in a single sport (Paillard, 2002; Kruger, 2004; Shaw, 2008; Matsuda, 2010; Paillard, 2006), or different groups of adult athletes competing in different sports (Davlin, 2004; Perrin, 2002; Bressel, 2007), trying to find a recognizable balance pattern characteristic of the specific sport activity. Few works focus on the evaluation of static and dynamic balance of adolescent athletes (Stepinski, 2003; Emery, 2005; Cowley, 2006; Gualtieri, 2008), while very few authors investigated the influence of different sport activities on the static and dynamic balance of children (Ricotti \& Ravaschio, 2011).

The importance of balance monitoring in adult athletes clearly appears from the conclusions of both articles by (Paillard, 2002) and (Paillard, 2006), the first one regarding judoists, and the second one regarding soccer players balance analysis. Results show that no differences in static balance are evident for judoists performing at regional level and judoists performing at national and international level. However, visual information seems to be more important for the higher level athletes, suggesting that the level of competition influences the sensory canals involved in balance. Static and dynamic balance performances are significantly different for soccer players involved in regional or national activities, suggesting that higher level players possess a greater sensitivity of sensory receptors or better integration of information than regional players. Balance performance analyses of athletes can be useful to investigate the role of specific factors on stance control. (Shaw, 2008) analyzed ten female volleyball players during jump-landing tasks, evaluating the efficacy of different bracing conditions in optimizing dynamic balance indexes, even in presence of fatigue. Preventing injuries is one of the main objectives of postural evaluation and correction. Kruger et al. analyzed 30 uninjured rugby players, finding that the application of a prophylactic knee brace significantly improved their proprioception performances.

It is also interesting to discover how and how much different sport activities lead to different balance performances and strategies. (Bressel, 2007) compared female athletes competing in soccer, basketball and gymnastics, finding substantial different static and dynamic performances among the groups. Similar conclusions were reported by Davlin, while (Perrin, 2002) focused on the differences between judo and dance activity, concerning balance control abilities. They found that, with eyes open, both judoists and dancers showed better static and dynamic stance than a control group not involved in any sport activity, while with eyes closed, only judoists have a significant better stance. This indirectly demonstrated that the two sport activities stimulate different proprioceptive canals.

An exhaustive review regarding adult athletes and the connection between their balance abilities and athletic performances is given by (Hrysomallis, 2011), describing that gymnasts tend to have the best balance ability, followed by soccer players, swimmers, active control subjects, and then basketball players. In (Hrysomallis, 2011) are also reported data regarding other sports, such as rifle shooting, golf, alpine skiing, surfing, judo, ice hockey, skating, and baseball. 
In (Stepinski, 2003) are reported data regarding the motor abilities of adolescent soccer players, comparing them with a control group composed by same age boys performing no sport activities. While speed, anaerobic power and frequency of movements are positively affected by soccer activity, smaller effects were found regarding coordination. This suggests that coordination training is often neglected in young soccer players, a negative factor that will influence their future performance. Balance is not only important for the execution of complex technical gestures, but it is also connected to the overall athlete's strength, as reported by (Cowley, 2006) and it can be connected to injury risks, as discussed by (Emery, 2005).

All these findings lead to the starting point, related to the importance of training children taking into account specific age-related balance training sessions, in order to induce effective changes in both sensory and motor systems influencing present and future balance performances. In this framework (Ricotti and Ravaschio, 2011) showed how dance can induce a strong improvement of static balance performances on 9 years-old soccer players during an overall period of six months. Being of particular significance, we report the results shown in the article (Figure 1).

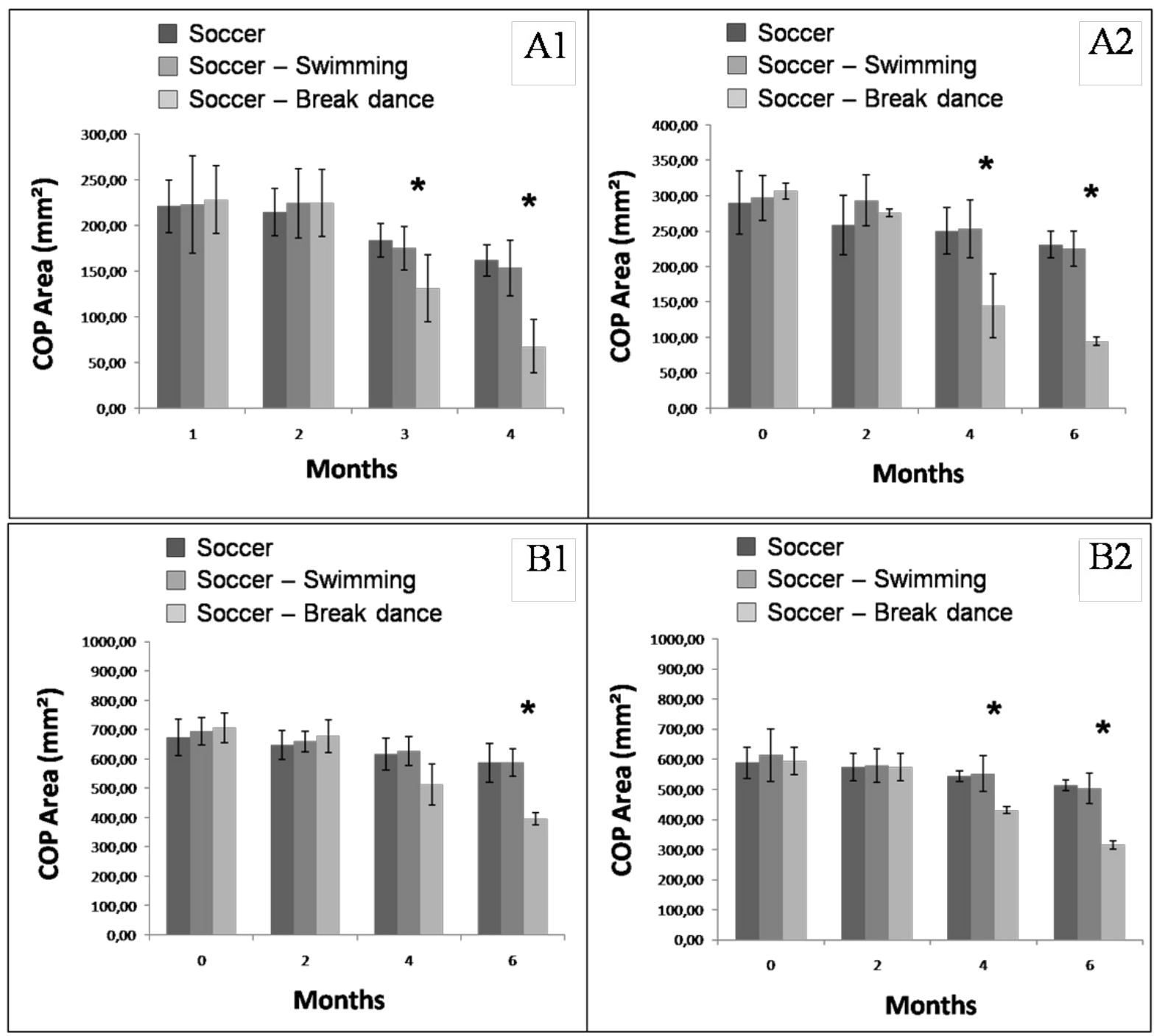

Figure 1. COP area recordings for three groups of young athletes (soccer players, soccer players also performing dance activity, and soccer players also performing swimming activity) during a six months evaluation and for four different static balance test conditions: double leg stance with eyes open (A1), double leg stance with eyes closed (A2), single leg stance on dominant limb (B1) and single leg stance on non-dominant limb (B2). ${ }^{*} p<0.05$. 
Only static balance tests were performed: double leg stance with eyes open (Figure 1(A1)), double leg stance with eyes closed (Figure 1(A2)), single leg stance on dominant limb (Figure 1(B1)) and single leg stance on non-dominant limb (Figure 1(B2)). The changes in balance abilities were found to be induced by the specific dance-related training movements in all the four conditions, and not by the simple addition of generic sport activity (as demonstrated by the comparison with a control group of swimmers). No findings have been reported regarding dynamic balance, raising the need of further research on such topic.

\section{CONCLUSION}

In order to create good adult athletes, it is of primary importance the attention that is paid to a complete and harmonic development of motor abilities at early ages, above all concerning the specific agerelated ones in each phase of the athlete's body development. Presently, there is poor attention on such aspects, not only in practical training sessions, but also concerning the existing literature. Few authors concentrated on the possible strategies to improve children static and dynamic balance, and on the influence that different sport activities have on postural strategies at early ages. It is desirable that future researches will focus on such aspects, in order to clarify the underlying mechanisms and to develop new effective strategies for the expression of athletes' potential.

\section{REFERENCES}

1. ARNOLD BL, SCHMITZ RJ. Examination of balance measures produced by the Biodex Stability System. Journal of Athletic Training. 1998; 33:323-327. [Abstract] [Back to text]

2. ASHTON-MILLER JA, WOJTYS EM, HUSTON LJ, FRY-WELCH D. Can proprioception really be improved by exercises? Knee Surgery, Sports Traumatology, Arthroscopy. 2001; 9:128-136. doi:10.1007/s001670100208 [Back to text]

3. ASSAIANTE C. Development of locomotor balance control in healthy children. Neuroscience and Biobehavioral Reviews. 1998; 22:527-532. doi:10.1016/S0149-7634(97)00040-7 [Back to text]

4. BALTER SGT, STOKROOS RJ, AKKERMANS E, KINGMA H. Habituation to galvanic vestibular stimulation for analysis of postural control abilities in gymnasts. Neuroscience Letters. 2004; 366:71-75. doi:10.1016/j.neulet.2004.05.015 [Back to text]

5. BOOHER J, THIBODEAU G. Athletic injury assessment. St. Louis, MO: Times Mirror/Mosby College Publishing; 1995. [Back to text]

6. BRESSEL E, YONKER JC, KRAS J, HEATH EM. Comparison of static and dynamic balance in female collegiate soccer, basketball, and gymnastics athletes. Journal of Athletic Training. 2007; 42:42-46. [Abstract] [Back to text]

7. CAMBIER D, COOLS A, DANNEELS L, WITVROUW E. Reference data for 4- and 5-year-old children on the Balance Master: values and clinical feasibility. European Journal of Pediatrics. 2001; 160:317-322. doi:10.1007/PL00008438 [Back to text]

8. COLBY SM, HINTERMEISTER RA, TORRY MR, STEADMAN JR. Lower limb stability with ACL impairment. Journal of Orthopaedic \& Sports Physical Therapy. 1999; 29:444-451. [Abstract] [Back to text]

9. COWLEY HR, FORD KR, MYER GD, KERNOZEK TW, HEWETT TE. Differences in neuromuscular strategies between landing and cutting tasks in female basketball and soccer athletes. Journal of Athletic Training. 2006; 41: 67-73. [Abstract] [Back to text]

10. DAVLIN CD. Dynamic balance in high level athletes. Perceptual and Motor Skills. 2004; 98: 1171-1176. doi:10.2466/pms.98.3c.1171-1176 [Back to text]

11. DICHGANS J, MAURITZ KH, ALLUM JH, BRANDT T. Postural sway in normals and atactic patients: analysis of the stabilizing and destabilizing effects of vision. Agressologie. 1976; 17, 15-24. [Abstract] [Back to text] 
12. DONKER SF, LEDEBT A, ROERDINK M, SAVELSBERGH GJ, BEEK PJ. Children with cerebral palsy exhibit greater and more regular postural sway than tipically developing children. Experimental Brain Research. 2008; 184: 363-370. doi:10.1007/s00221-007-1105-y [Back to text]

13. EKHDAHL C, JARNLO GB, ANDERSSON SI. Standing balance in healthy subjects. Evaluation of a quantitative test battery on a force platform. Scandinavian Journal of Rehabilitation Medicine. 1989; 21: 187-195. [Abstract] [Back to text]

14. EMERY CA. Is there a clinical standing balance measurement appropriate for use in sports medicine? A review of the literature. Journal of Science and Medicine in Sports. 2003; 6:492504. doi:10.1016/S1440-2440(03)80274-8 [Back to text]

15. EMERY CA, MEEUWISSE WH, HARTMANN SE. Evaluation of risk factors for injury in adolescent soccer. American Journal of Sports Medicine. 2005; 33:1882-1891. doi: 10.1177/0363546505279576 [Back to text]

16. ERA P, SCHROLL M, YTTING H, GAUSE-NILSSON I, HEIKKINEN E, STEEN B. Postural balance and its sensory-motor correlates in 75-year-old men and women: a cross-national comparative study. The Journals of Gerontology Series A: Biological Sciences and Medical Sciences. 1996; 51A: M53-M63. doi:10.1093/gerona/51A.2.M53 [Back to text]

17. FREITAG CM, KLESER C, SCHNEIDER M, VON GONTARD A. Quantitative assessment of neuromotor function in adolescents with high functioning autism and Asperger syndrome. Journal of Autism and Developmental Disorders. 2007; 37:948-959. doi:10.1007/s10803-0060235-6 [Back to text]

18. GELDHOF E, CARDON G, DE BOURDEAUDHUIJ I, DANNEELS L, COOREVITS P, VANDERSTRAETEN G, DE CLERCQ D. Static and dynamic standing balance: test-retest reliability and reference values in 9 to 10 year old children. European Journal of Pediatrics. 2006; 165: 779-786. doi:10.1007/s00431-006-0173-5 [Back to text]

19. GOULDINGA A, JONESA IE, TAYLOR RW, PIGGOT JM, TAYLOR D. Dynamic and static tests of balance and postural sway in boys: effects of previous wrist bone fractures and high adiposity. Gait \& Posture. 2003; 17:136-141. doi:10.1016/S0966-6362(02)00161-3 [Back to text]

20. GRAYBIEL A, FREGLY A. A new quantitative ataxia test battery. Acta oto-laryngologica. 1966; 61:292-321. doi:10.3109/00016486609127066 [Back to text]

21. GRIBBLE PA, HERTEL J. Considerations for normalizing measures of the Star Excursion Balance Test. Measurement in Physical Education and Exercise Science. 1999; 7:89-100. doi:10.1207/S15327841MPEE0702_3 [Back to text]

22. GRIGG P. Peripheral neural mechanisms in proprioception. Journal of Sport Rehabilitation. 1994; 3:2-17. [Abstract] [Back to text]

23. GRIFFIN LY, AGEL J, ALBOHM MJ, ARENDT EA, DICK RW, GARRETT WE, GARRICK JG, HEWETT TE, HUSTON L, IRELAND ML, JOHNSON RJ, KIBLER WB, LEPHART S, LEWIS JL, LINDENFELD TN, MANDELBAUM BR, MARCHAK P, TEITZ CC, WOJTYS EM. Noncontact anterior cruciate ligament injuries: risk factors and prevention strategies. Journal of the American Academy of Orthopaedic Surgeons. 2000; 8:141-150. [Abstract] [Back to text]

24. GUALTIERI D, CATTANEO A, SARCINELLA R, CIMADORO G, ALBERTI G. Relationship between balance capacity and jump ability in amateur soccer players of different ages. Sport Sciences for Health. 2008; 3:73-76. doi:10.1007/s11332-008-0074-2 [Back to text]

25. GURFINKEL VS. Muscle afferentation and postural control in man. Agressologie. 1973; 14:1-8. [Back to text]

26. GUSKIEWICZ KM, PERRIN DH. Research and clinical applications of assessing balance. Journal of Sport Rehabilitation. 1996; 5: 45-63. [Abstract] [Back to text]

27. HARRISON AL. The influence of pathology, pain, balance, and self-efficacy on function in women with osteoarthritis of the knee. Physical Therapy. 1994; 84: 822-831. [Abstract] [Back to text] 
28. HATZITAKI V, ZISI V, KOLLIAS I, KIOUMOURTZOGLOU E. Perceptual-motor contributions to static and dynamic balance control in children. Journal of Motor Behavior. 2002; 34:161-170. doi:10.1080/00222890209601938 [Back to text]

29. HILL KD, BERNHARDT J, MCGANN AM, MALTESE D, BERKOVITS D. A new test of dynamic standing balance for stroke patients: reliability, validity and comparison with healthy elderly. Physiotherapy Canada. 1996; 1:257-262. doi:10.3138/ptc.48.4.257 [Back to text]

30. HRYSOMALLIS C. Balance abilities and athletic performances. Sports Medicine. 2011; 41:221232. doi:10.2165/11538560-000000000-00000 [Back to text]

31. HUXHAM FE, GOLDIE PA, PATLA AE. Theoretical considerations in balance assessment. Australian Journal of Physiotherapy. 2001; 47:89-100. [Full Text] [Back to text]

32. HYTÖNEN M, PYYKKÖ I, AALTO H, STARK J. Postural control and age. Acta otolaryngologica. 1993; 113:119-122. doi:10.3109/00016489309135778 [Back to text]

33. ISHIZAKI H, PYYKKÖ I, AALTO H, STARCK J. Repeatability and effect of instruction of body sway. Acta oto-laryngologica. 1991; 111:589-592. doi:10.3109/00016489109131478 [Back to text]

34. IVERSON BD, GOSSMAN MR, SHADDEAU SA, TURNER ME. Balance performance, force production, and activity levels in noninstitutionalized men 60 to 90 years of age. Physical Therapy. 1990; 70: 348-355. [Abstract] [Back to text]

35. JUNTUNEN J, MATIKAINEN E, YLIKOSKI J, YLIKOSKI M, OJALA M, VAHERI E. Postural body sway and exposure to high-energy impulse noise. The Lancet. 1987; 330:261-264. doi:10.1016/S0140-6736(87)90840-3 [Back to text]

36. KINNEY LAPIER T, LIDDLE S, BAIN C. A comparison of static and dynamic standing balance in older men versus women. Canadian Physiotherapy.1997; 49: 207-213. [Back to text]

37. KIRSCHENBAUM N, RIACH CL, STARKES JL. Non-linear development of postural control and strategy use in young children: a longitudinal study. Experimental Brain Research. 2001; 140:420-431. doi:10.1007/s002210100835 [Back to text]

38. KOWULSKI K, DI FABIO RP. Gross motor and balance impairments in children and adolescents with epilepsy. Developmental Medicine \& Child Neurology. 1995; 37:604-619. doi:10.1111/j.1469-8749.1995.tb12049.x [Back to text]

39. KRUGER TH, COETSEE MF, DAVIES S. The effect of prophylactic knee bracing on proprioception performance in first division rugby union players. Sports Medicine. 2004; 16:3336. [Back to text]

40. LEBIEDOWSKA MK, SYCZEWSKA M. Invariant sway properties in children. Gait \& Posture. 2000; 12:200-204. doi:10.1016/S0966-6362(00)00080-1 [Back to text]

41. LEE HY, CHERNG RJ, LIN CH. Development of a virtual reality environment for somatosensory and perceptual stimulation in the balance assessment of children. Computers in Biology and Medicine. 2004; 34:719-733. doi:10.1016/j.compbiomed.2003.10.004 [Back to text]

42. MAKI BE. Selection of perturbation parameters for identification of the posture-control system. Medical and Biological Engineering and Computing. 1986; 24: 561-568. doi:10.1007/BF02446257 [Back to text]

43. MALLAU S, BOLLINI G, JOUVE JL, ASSAIANTE C. Locomotor skills and balance strategies in adolescents idiopathic scoliosis. Spine. 2007; 32:E14-E22. doi:10.1097/01.brs.0000251069.58498.eb [Back to text]

44. MATSUDA S, DEMURA S, NAGASAWA Y. Static one-legged balance in soccer players during use of a lifted leg. Perceptual and Motor Skills. 2010; 111:167-177. doi:10.2466/05.23.26.27.PMS.111.4.167-177 [Back to text]

45. MCKINLEY P, PEDOTTI A. Motor strategies in landing from a jump: the role of skill in task execution. Experimental Brain Research. 1992; 90:427-440. doi:10.1007/BF00227257 [Back to text] 
46. MORASSO PG, SPADA G, CAPRA R. Computing the COM from the COP in postural sway movements. Human Movement Science. 1999; 18:759-767. doi:10.1016/S01679457(99)00039-1 [Back to text]

47. NAGY E, TOTH K, JANOSITZ G, KOVACS G, FEHER-KISS A, ANGYAN L, HORVAT G. Postural control in athletes participating in an ironman triathlon. The European Physical Journal - Applied Physics. 2004; 92:407-413. doi:10.1007/s00421-004-1157-7 [Back to text]

48. NASHER LM, BLACK FO, WALL C. Adaptation to altered support and visual conditions during stance: patients with vestibular deficits. Journal of Neurosciences. 1982; 2:536-544. [Abstract] [Back to text]

49. NJIOKIKTJIEN C, DE RIJKE W, DIEKER-VAN OPHEM A, VOORHOEVE-COEBERGH O. A possible contribution of stabilography to the different diagnosis of cerebellar processes. Agressologie. 1978; 19: 87-88. [Abstract] [Back to text]

50. NOLAN L, GRIGORENKO A, THORSTENSSON A. Balance control: sex and age differences in 9- to 16-year-olds. Developmental Medicine \& Child Neurology.2005; 47:449-454. doi:10.1017/S0012162205000873 [Back to text]

51. PAILLARD T, COSTES-SALON C, LAFONT C, DUPUI P. Are there differences in postural regulation according to the level of competition in judoists? British Journal of Sports Medicine. 2002; 36:304-305. doi:10.1136/bjsm.36.4.304 [Back to text]

52. PAILLARD T, NOÉ F, RIVIĖRE T, MARION V, MONTOYA R, DUPUI P. Postural performance and strategy in the unipedal stance of soccer players at different levels of competition. Journal of Athletic Training. 2006; 41:172-176. [Abstract] [Back to text]

53. PALMIERI RM, INGERSOLL CD, STONE MB, KRAUSE BA. Center-of-.pressure parameters used in the assessment of postural control. Journal of Sports and Rehabilitation. 2002; 11:5166. [Abstract] [Back to text]

54. PERRIN P, DEVITERNE D, HUGEL F, PERROT C. Judo, better than dance, develops sensorimotors adaptabilities involved in balance control. Gait \& Posture. 2002; 15:187-194. doi:10.1016/S0966-6362(01)00149-7 [Back to text]

55. PETERKA RJ. Sensorimotor integration in human postural control. Journal of Neurophysiology. 2002; 88:1097-1118. [Abstract] [Back to text]

56. RIBADI H, RIDER RA, TOOLE T. A comparison of static and dynamic balance in congenitally blind, sighted, and sighted blindfolded adolescents. Adapted Physical Activity Quarterly. 1987; 4:220-225. [Abstract] [Back to text]

57. RICOTTI L, RAVASCHIO A. Break dance significantly increases static balance in 9 years-old soccer players. Gait \& Posture. 2011; 33:462-465. doi:10.1016/j.gaitpost.2010.12.026 [Back to text]

58. RIEMANN BL, GUSKIEWICZ KM, SHIELDS EW. Relationship between clinical and forceplate measures of postural stability. Journal of Sport Rehabilitation. 1999; 8:71-82. [Abstract] [Back to text]

59. RIVAL C, CEYTE H, OLIVIER I. Developmental changes of static standing balance in children. Neuroscience Letters. 2005; 376:133-136. doi:10.1016/j.neulet.2004.11.042 [Back to text]

60. SCHMID M, CONFORTO S, LOPEZ L, RENZI P, D'ALESSIO T. The development of postural strategies in children: a factorial design study. Journal of NeuroEngineering and Rehabilitation. 2005; 30:29-39. doi:10.1186/1743-0003-2-29 [Back to text]

61. SHAW MY, GRIBBLE PA, FRYE JL. Ankle bracing, fatigue, and time to stabilization in collegiate volleyball athletes. Journal of Athletic Training. 2008; 43:164-171. doi:10.4085/10626050-43.2.164 [Back to text]

62. STEPINSKI M, ZWIERKO T, FLORKIEWICZ B, DEBICKA J. The level of the chosen motor abilities of 13 years old soccer players. Journal of Human Kinetics. 2003; 9:1-8. [Abstract] [Back to text]

63. WEINECK J. Optimales training. Verlag: GmbH; 2001. [Back to text] 
64. WIKSTROM EA, POWERS ME, TILLMAN MD. Dynamic stabilization time after isokinetic and functional fatigue. Journal of Athletic Training. 2004; 39: 247-253. [Abstract] [Back to text]

65. WIKSTROM EA, TILLMAN MD, SMITH AN, BORSA PA. A new force-plate technology measure of dynamic postural stability: the Dynamic Postural Stability Index. Journal of Athletic Training. 2005; 40:305-309. [Abstract] [Back to text]

66. WINTER DA, PATLA AE, FRANK JS. Assessment of balance control in humans. Medical Progress through Technology. 1990; 16:31-51. [Full Text] [Back to text] 